\title{
Consumo de productos infantiles en la base de la pirámide poblacional: análisis de los mecanismos influyentes ${ }^{1}$
}

\section{Products consumption in children at the base of the population pyramid: analysis of influential mechanisms}

DOI: http://dx.doi.org/10.17981/econcuc.38.1.08

\author{
María Cristina Otero Gómez ${ }^{2}$ \\ Wilson Giraldo Pérez ${ }^{3}$
}

\begin{abstract}
Resumen
La base de la pirámide poblacional representa uno de los mayores mercados en el mundo, por tanto, se convierte en una oportunidad para transformar la pobreza en parte de la solución mediante el diseño de productos que suplan las necesidades y aspiraciones de los miembros que conforman este segmento. Por lo cual, se define el objetivo de identificar los mecanismos de influencia que intervienen en la toma de decisiones de consumo en los infantes entre 5 y 9 años de la base de la pirámide de Villavicencio, Colombia. Se establece el método cualitativo y se selecciona la técnica de observación no participante y las entrevistas a profundidad, las cuales fueron realizadas a nueve menores de edad. Los hallazgos ponen en evidencia tres grupos de influencias organizados: influenciadores fuertes, entre los que se encuentran los semejantes etarios y el precio de los productos; influenciadores moderados, representados en las marcas diseñadas para este segmento; e influenciadores menores, soportados en el control parental. Se concluyó que las escuelas y las tiendas de barrio son los puntos de venta donde mayor influencia recibe el segmento de mercado correspondiente al consumidor infantil de la base de la pirámide.
\end{abstract}

Palabras clave: segmentación de mercados; productos infantiles; consumidor infantil; base de la pirámide; influencia de marcas.

Recibido: 20/3/2017 Devuelto para revisión: 19/5/17 Aceptado: 24/5/2017.

${ }^{1}$ Artículo resultados de la investigación titulada "El consumo infantil en la base de la pirámide de Villavicencio (Colombia) financiado por la Universidad de los Llanos con el código C01-F05-002-2016, iniciado en febrero de 2016 y finalizado en Enero de 2017. Se circunscribe al Grupo de Investigación Dinámicas de Consumo de la Facultad de Ciencias Económicas. Línea de investigación Crecimiento y Desarrollo Socioeconómico Orinoquense.

${ }^{2}$ Cursante del Doctorado en Marketing. Magíster en Mercadeo. Docente y Directora del Grupo de Investigación Dinámicas de Consumo de la Facultad de Ciencias Económicas de la Universidad de los Llanos. Villavicencio-Colombia. Correo electrónico: motero@unillanos.edu.co

${ }^{3}$ Cursante del Doctorado en Marketing. Magíster en Mercadeo. Docente de la Facultad de Ciencias Económicas de la Universidad de los Llanos, Villavicencio-Colombia. Correo electrónico wgiraldo@unillanos.edu.co

\footnotetext{
- The author; licensee Universidad de la Costa - CUC.
} Económicas CUC vol. 38 no. 1, pp. 165-184. Enero - Junio, 2017 Barranquilla. ISSN 0120-3932 Impreso, ISSN 2382-3860 Online

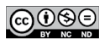




\begin{abstract}
The present Work is aimed to illustrate the base of the population pyramid as one of the largest markets in the world, therefore, it becomes an opportunity to transform poverty into a part of the solution by designing products that fulfil the needs and aspirations of the members of the mentioned segment of the society. Therefore, the objective of identifying the influential mechanisms related to making consumption decisions in children over 5 and 9 years of age at the base of the pyramid in Villavicencio, Colombia, was defined. With regard to the research methodology a qualitative method as well techniques of non-participant observation and in-depth interviews were used, the sample chosen in this research work were nine infant. The obtained results allowed to classify three groups of organized influencers. The first group was the macro influencers, there have existed not only similar peers but also the price of products; the second group was represented by the moderate influencers, these ones have been related to the brands designed for this segment, the third group was the minor influencers which were supported by the parental control. Hence, taking into account the results of this work it can be said that schools and neighborhoods stores are the key purchase influencers to the consumption in children at the base of the pyramid.
\end{abstract}

Keywords: market segmentation, children's products, infant consumer, base of the pyramid, brand influences.

\title{
Introducción
}

El consumo es un fenómeno de los seres vivos que tiene diferentes análisis soportados en factores económicos, sociales y culturales. Desde las ciencias económicas este es visto como la etapa final del ciclo económico, definido como el momento en que un bien o servicio produce alguna utilidad al sujeto consumidor. A partir del concepto del marketing social, el consumo debe ser responsable a nivel social y ambiental, debe cubrir las necesidades actuales de la oferta y la demanda, pero que al mismo tiempo conserve o mejore la capacidad de generaciones futuras para cubrir sus necesidades (Kotler \& Armstrong, 2012, p. 11).

La nueva generación infantil del siglo XXI, es única en la historia, pues los niños han estado conectados al mundo digital desde temprana edad debido a la penetración de la tecnolo- 
gía. Estos infantes se caracterizan por ser más directos en sus preguntas a los adultos, en buscar las respuestas por si mismos a través de foros y chats de preguntas por Internet, y también por minimizar el tiempo de espera para sus respuestas. "Si hay un mercado donde los cambios se producen sin cesar, el consumidor evoluciona trepidantemente y las necesidades o deseos están mutando de forma vírica, éste es, sin lugar a dudas, el de los niños” (Tur \& Ramos, 2008, p. 11).

El consumo es una acción que se da forma natural, sin importar la condición económica de los miembros de la sociedad, sin embargo esto se hace viable en función del poder adquisitivo de cada individuo. Se considera que mientras mayor sea la disponibilidad de efectivo de los consumidores de la clase alta, acceden con mayor frecuencia a las compras sin recurrir a créditos, la clase media lo hace a través de créditos de consumo con altas tasas de interés dentro del sistema bancario. Por su parte, los consumidores de las clases menos favorecidas, en la mayoría de casos recurren a créditos no regulados por la banca, lo que encarece el pago final de los productos que finalmente consumen.

De esta forma, entender la base de la pirámide poblacional es a la vez un reto y una oportunidad para el mundo empresarial y de los negocios. Este segmento posee un comportamiento de consumo similar a la de otros niveles socioeconómicos, pero difiere de ellos en su cultura de consumo y en la disponibilidad financiera. Esta última, generalmente se relaciona con el presupuesto financiero diario, el cual cubre y atiende el consumo de bienes y servicios en pequeñas cantidades.

Adicionalmente, el consumidor de la base de la pirámide al igual que los demás consumidores está expuesto a múltiples factores que promueven el consumo. En particular, el consumidor infantil no es ajeno a los cambios presentados en las telecomunicaciones, en la estructura familiar, en el rol de los padres y/o en los modelos pedagógicos. De este modo, las influencias del entorno moldean y condicionan su conducta como consumidor, la cual por su edad se encuentra aún en formación; por lo que es necesario identificar ¿cuáles son los mecanismos influenciadores que intervienen en sus decisiones de consumo?

En este sentido, el presente artículo hace referencia a cuatro mecanismos que ejercen influencia en el consumidor infantil como son: 1) la marca y sus elementos visuales; 2) el dinero expresado en el precio del producto; 3) la relación con los adultos y 4) la relación con sus semejantes. Por ello, en su estructura presenta una revisión teórica y conceptual desde 
los campos científico y empresarial. Posteriormente, se explica la metodología empleada en la investigación, junto con la presentación detallada de los resultados para cada uno de los mecanismos influenciadores estudiados. Por último, se describen las conclusiones y las limitaciones presentadas en el estudio, así como el planteamiento de una futura línea de investigación.

\section{El consumidor infantil}

Como lo manifestaban Tur y Ramos, el mercado infantil ha evolucionado trepidantemente, pero surge el interrogante ¿basado en qué estado inicial se pueden analizar estos cambios? La revisión de literatura permite establecer que son pocos los trabajos que han analizado la evolución en el estudio del comportamiento del consumidor infantil como temática de investigación.

No obstante, se destaca el trabajo de Cook (2009) quien presenta una panorámica completa desde principios del siglo XX hasta la actualidad. Este autor plantea que existen 6 épocas claramente diferenciadas en relación con los estudios sobre el consumidor infantil, así: 1) el momento introductorio circunscrito al análisis empresarial el cual se ubica entre 1910 y $1950 ; 2)$ Los estudios se realizan desde el punto de vista académico entre los años 1950 y 1970; 3) Surge la producción científica entre 1970 y 1980. Para esta época sobresalieron dos planteamientos en los cuales a los niños se les asigna el estatus de consumidor. Por un lado, "los niños son, de hecho, los consumidores y, de hecho, constituyen un mercado", y por otro, "los productos se imponen a los niños por su cultura como el medio principal para satisfacer sus necesidades" (Mc Neal, 1987, p.p. 25 y 90). 4) Aparece el paradigma cualitativo interpretativo entre 1980 y 1990; 5) Marca el final del siglo XX entre los años 1990 y 2000, donde las investigaciones se orientan a las ciencias empresariales, puesto que los estudios previos se relacionaron con las ciencias sociales y humanas; 6) Se caracterizó por el interés en el comportamiento infantil en torno a las prácticas cotidianas de consumo; el uso de los bienes y los medios de comunicación; comportamiento en los entornos comerciales; y el análisis del simbolismo representado en las marca y la relación con las celebridades.

Los periodos anteriores están enmarcados en un entorno mundial y para el caso de Colombia los resultados son aún más escasos, sin embargo, se destaca el estudio de Amar, Llanos, 
Abello \& Denegri (2003) titulado "Desarrollo del pensamiento económico en niños de la región caribe colombiana" el cual buscó profundizar en el desarrollo de la comprensión de conceptos económicos. Junto a ellos, se hace presente el aporte de Silva (2009) con el estudio "El consumo, ¿un juego de niños?” aplicado en Bogotá en el cual se trató el tema del consumo simbólico en la configuración de estilos de vida de los tweens. En un contexto local, se encuentra el aporte realizado por Giraldo \& Otero (2015) con la publicación denominada "El consumidor infantil en Villavicencio, Colombia", donde se pudo establecer que el entorno social y las prácticas culturales de cada familia son determinantes para el comportamiento del consumidor infantil.

Debido a la escasez de estudios empíricos contextualizados a la realidad colombiana, este artículo aporta en la construcción de literatura sobre las influencias del entorno del consumidor infantil en la base de la pirámide. Para nadie es un secreto que se trata de un nicho con un gran potencial para el desarrollo de negocios, por ello, tal como afirma Prahalad (2005), "Si dejamos de pensar en los pobres como víctimas o como carga, y empezamos a reconocerlos como empresarios creativos y con capacidad de recuperación, y como consumidores con sentido del valor, se abrirá un mundo de nuevas oportunidades" (p. 1). Por consiguiente, las empresas en su gestión comercial deben establecer estrategias de marketing que no solamente tengan como finalidad la creación de bienes y servicios que conlleven al cumplimiento de indicadores económicos, también deben ajustarse a las características de los diferentes segmentos, de tal forma que generen la satisfacción de los deseos de sus consumidores.

Desde esta perspectiva, se destaca la importancia del marketing estratégico adaptado a nuevos paradigmas de competitividad, donde las empresas deben responder con inmediatez ante los cambios del entorno. En este sentido, como afirman Mendoza y López (2015) es necesario pensar estratégicamente, lo que implica "salir de los sistemas convencionales, tener la capacidad analítica, ser deductivo, poseer alta dosis de creatividad, ser intuitivo, tener gran capacidad imaginativa, no ser lineal, ser innovador y descubrir estrategias novedosas" (p. 164). Estos elementos son necesarios para adecuarse al medio ambiente y a las necesidades del mercado, por ello, el éxito de la estrategia depende de la capacidad de la empresa para orientar sus esfuerzos al cliente. Así pues, no basta con analizar variables cuantitativas que midan solamente la calidad y el precio, es indispensable adicionar un "valor" al consumidor que garantice la sostenibilidad de la empresa en el mercado. 
En este orden de ideas, para que las empresas puedan responder adecuadamente al consumidor infantil "precisan de una mayor comprensión de este mercado no solo al considerar el estudio del desarrollo cognitivo en las decisiones de consumo, sino también teniendo en cuenta el contexto social y cultural" (Silva, 2009, p.81), esto significa que las estrategias de la empresa se definen en función del consumidor. De este modo, las organizaciones evidencian su condición de sistema social al desarrollar productos valorados por los clientes, y para el caso, se trata de un segmento que reúne dos condiciones sensibles como son la infancia y la limitación del gasto. En Colombia, empresas como Alpina han comprendido la importancia de llevar productos a la población con menores recursos, así lo estableció su Vicepresidente de Mercadeo al afirmar que "La oportunidad del mercadeo en los estratos de menores ingresos está abierta, pero es indispensable innovar para llegar con los productos adecuados" (Fernández, 2008). Autores como Villalba, Hurtado, Guarín y Casas (2013) sostienen que "la innovación aporta a que los productos y servicios tengan siempre valor" (p. 25); este valor también es percibido desde edades muy tempranas. De este modo, el consumidor infantil visualiza el consumo de determinados productos como una forma de jerarquización social donde el dinero y las marcas les permiten por un lado, distinguirse entre sus pares, y por otro, son una demostración de pertenencia a una comunidad que comparte valores y patrones similares de consumo.

\section{Metodología}

Las características de la investigación motivan la utilización del paradigma relativista como base epistemológica, el cual busca conocer e interpretar el comportamiento de los seres humanos desde una perspectiva más integral que descriptiva. De esta forma, se desarrolló una investigación cualitativa en la cual se asumió la propuesta de Creswell (1994) quien plantea que el investigador actúa desde lo epistémico con el objeto de investigación; desde lo axiológico con la realidad influencia por valores de cada sujeto expresados en el lenguaje no verbal; y desde la perspectiva metodológica por el proceso inductivo, donde su diseño es emergente. $\mathrm{Su}$ veracidad y confiabilidad se hacen a través de la verificación de la generalización de la información obtenida de los datos, y no de la validez interna de un instrumento. En este sentido, la presente investigación se soporta en lo que Báez (2012) denomina la "longevidad" de los resultados, puesto que "las motivaciones, las actitudes, las creencias, los juicios, etcétera tienen tendencia a permanecer estables a lo largo de periodos muy prolongados" (p.24). 


\section{Trabajo de campo}

Para la selección de la muestra, se consideró que por tratarse de un grupo poblacional altamente sensible como son los infantes de 5 a 9 años de la base de la pirámide en la ciudad de Villavicencio, y para dar cumplimiento a los protocolos éticos de investigaciones con menores de edad, la investigación se realizó con la mediación de una institución educativa. El proceso inicial se llevó a través del rector, quien envió una solicitud de permiso a los padres o acudientes de cada niño para que hicieran parte integral y fundamental de la investigación. Por tanto, solamente se seleccionaron como integrantes de la muestra aquellos infantes a quienes sus padres autorizaron, y en los análisis finales se omiten nombres o características que permitan su identificación, siendo en total 9 infantes de género masculino y femenino quienes participaron como informantes.

Lo relacionado con la recopilación de los datos se utilizaron varias técnicas que permitieron una visión comprensiva del comportamiento de estos consumidores infantiles, el proceso fue:

- Adecuación de un espacio para la realización de la simulación de compra. En este espacio se dispusieron tiendas especializadas en productos de consumo.

- La realización de grupos focales en donde se indagó sobre varios temas en general, pero con orientación hacia la influencia del dinero.

- Presentación de un reel de comerciales para que los niños estuvieran expuestos a algunas marcas que se encontraban disponibles en las tiendas dispuestas para la compra.

- Observación no participante, la cual consistió en el desarrollo de una compra simulada, donde cada niño tuvo disponible COP50.0004. Las compras se realizaron en 4 tiendas simuladas y especializadas en la venta de productos de: aseo, golosinas, comida saludable y snacks.

- Posterior a la compra simulada se realizó una entrevista a profundidad a cada consumidor con el fin de indagar y comprender las razones de la compra.

\footnotetext{
${ }^{4}$ COP es la abreviación formal de la unidad monetaria de curso legal en Colombia según el estándar internacional ISO 4217
} 
Los resultados obtenidos en las entrevistas a profundidad se procesaron a través del software AtlasTi. Se analizaron las respuestas de los participantes, con el fin de formar un concepto general y una primera comprensión del fenómeno global, así como para detectar acuerdos y desacuerdos significativos entre los infantes de 5 a 9 años de la base de la pirámide de Villavicencio (Colombia). Adicionalmente se sistematizaron los hallazgos generalizables de la observación no participante obtenidos durante los momentos de la presentación del reel de comerciales y en la compra simulada realizada por los infantes.

\section{Variables de estudio}

Aunque los mecanismos que ejercen influencia en un consumidor provienen de múltiples orígenes, para la elaboración del presente artículo se hizo hincapié en cuatro variables, así: 1) La marca de los productos, como un elemento que puede facilitar la toma de decisiones sin mayor cantidad de información; 2) el precio del producto considerado un factor decisivo puesto que es el que facilita o impide la transacción de intercambio; 3 ) las relaciones con los adultos mediadas por el control parental en el que se encuentran inmersos los infantes y 4) las relaciones con sus pares etarios quienes pueden convertirse en un modelo a seguir o a evitar.

\section{Elemento integral del producto: la marca.}

La manera en que las empresas comunican un mensaje publicitario a los niños, puede generar un impacto inesperado en sus percepciones. Los niños crean asociaciones, y asignan significados positivos o negativos con marcas y productos, a través de su proceso de socialización (Reyes, 2013).

Aunque se pueda creer que este comportamiento corresponde al de niños pertenecientes a familias con ingresos altos o medios, existen estudios que reflejan el mismo efecto de asociación en los niños ubicados en la base de la pirámide. Tal es el caso de Chikweche \& Fletcher, (2011), quienes encontraron que estas personas reconocen y diferencian las marcas de los productos, conocen sus nombres, imágenes asociadas, y sus ventajas. 
En este sentido, la publicidad de una marca condiciona aspectos emocionales en los infantes cuando, por ejemplo, utilizan mascotas publicitarias o hacen exaltación de los atributos del producto asociados a la fuerza y al crecimiento que el consumidor obtiene al utilizarlos (Portela, 2011, p. 107). La extensión de lealtad de la marca a la que se hace referencia, entre los niños se basa en función de la interacción de dos elementos: la confianza y los estímulos del marketing. Tal lealtad resulta de la visibilidad diaria del producto mediante el uso de la marca a través de sus padres, también de la exposición de las marcas en los medios de comunicación, y en ambientes con otros niños incluso en la escuela, o los patrocinios (Paul, 2002).

La importancia de la marca y su influencia sobre los niños ha sido objeto de estudio en diferentes trabajos como los de Dotson \& Hyatt (1994), Roper \& Shah (2007) y Nairn, Griffin \& Wicks (2008). En su conjunto estos trabajos evidencian la vulnerabilidad del segmento infantil a la influencia marquista, quizás en mayor medida que en otro tipo de colectivos, debido a su inmadurez evolutiva. Sin embargo, esa vulnerabilidad puede estar condicionada por otros mecanismos como el tipo de patrón educativo que las familias hayan ejercido sobre los niños.

\section{Elemento de intercambio: el precio}

La racionalidad infantil fue estudiada por Mocan (2007), su análisis se sustenta desde la perspectiva de la asimetría existente entre la cantidad de información brindada a un mercado y la racionalidad utilizada por un consumidor en la toma de decisiones. El autor establece que durante la compra no se utiliza el total de la información disponible, sino que en ocasiones se realiza una selección adversa por parte de los consumidores.

Estos niños y jóvenes del siglo XXI nacidos durante los últimos 15 años, poseen más poder personal, más influencia y atención de los adultos, pero especialmente cada vez tendrán más dinero y capacidad de decisión en las compras tanto personales como familiares:

Los padres "premian a los niños" comprándoles cosas en la tienda si se "portan bien". Se registraron casos en que los padres iban a hacer una compra regular como la leche y el pan y terminaron comprando una colombina, o un paquete de pasabocas con un muñequito del superhéroe de moda en su interior. (Acevedo, 2005, p.32). 
En la presente investigación la racionalidad en los consumidores infantiles es analizada desde el constructo del intercambio económico. Autores como Delval \& Echeita (1991) afirman que los niños y niñas mayores de diez años se encuentran con una adecuada capacidad cognitiva y de memoria para diferenciar las denominaciones de la moneda y realizar operaciones matemáticas de adición y sustracción como conocimientos básicos para generar la comprensión del intercambio económico y ser considerados consumidores. De esta forma, se analiza la percepción del precio como elemento de la racionalidad y del intercambio económico y su relación con la decisión de compra de los niños.

\section{Control parental en las decisiones de consumo}

La familia ha sido estudiada como un factor influyente en distintos trabajos. Se destacan los aportes de Rummel, Howard, Swinton \& Seymour (2000) apoyados en la teoría psicológica con el concepto de reactancia. Estos autores han centrado su atención en clasificar las familias en función de los patrones generales de comunicación existentes en su seno. También se encuentran los postulados realizados por Herrera, Brito, Pérez, Martínez \& Díaz (2001) quienes afirman que son pocas las investigaciones en las que se consideran los estilos educativos del padre y la madre por separado. Habitualmente se habla de padres de forma genérica, sin tener en cuenta la diferente repercusión que puede tener en los hijos el estilo percibido por cada progenitor.

Diferentes investigaciones evidencian que existen tres agentes de socialización muy importantes que influyen en el comportamiento del consumidor infantil, para esta investigación se toman los propuestos por Moschis \& Moore (1979) los cuales son: padres, semejantes, y medios masivos de comunicación - principalmente la televisión -. De acuerdo con estos estudios, los padres tienen el papel de influencia dominante hasta que el niño llega a la adolescencia, momento en el cual los semejantes se convierten en la fuente predilecta de la información. Los padres son el origen principal de influencias sensatas sobre niños, los semejantes y medios de comunicación son principalmente la fuente de las influencias irracionales (Moschis \& Churchill, 1978). 


\section{Modelos a seguir: Los Semejantes}

En diferentes contextos la influencia de los semejantes es cambiante; por ejemplo, los niños que son cuidados por uno de sus padres después de la escuela probablemente irán de compras acompañado por este más a menudo que otros niños, de este modo, tendrán la oportunidad de aprender el comportamiento del progenitor, más que los comportamientos de sus iguales.

De otro lado, los niños de familias donde trabajan los dos padres, o en los casos donde existe la figura de padre o madre soltero (a), se manifiestan situaciones donde la influencia en el consumo la ejercen los familiares o los semejantes, debido al mayor tiempo de interacción con ellos en comparación con el tiempo de permanencia con sus padres. Wolf (1982) considera que los actos cotidianos se ven influenciados por su contexto, lenguaje, escena social y acción, los cuales a su vez están interconectados y presentan reciprocidad entre sí.

Finalmente, los niños que se quedan en la casa a solas hasta que sus padres regresan de los trabajos, tienen mayor interacción con su entorno social en general y son expuestos a más horas de contacto con los medios de comunicación y pueden ser más influidos por sus amigos que por otro tipo de personas (Dotson \& Hyatt, 2000).

\section{Resultados}

\section{Influencia de la Marca}

A pesar de que estos niños de 5 a 9 años de la base de la pirámide mostraron atención al momento de la exposición publicitaria de las marcas Gelatina Boggy, Pony Malta, Galletas Festival, Avena Alpina, Goma de mascar Bubbaloo, y cereales Kellogg's, su interés no se evidenció en el momento de la compra. Durante el reel los niños cantaron y bailaron las canciones que acompañaron los comerciales, lo que denota que las marcas presentan recordación en este segmento.

No obstante, todos los niños direccionan su compra inicial a la categoría de aseo, la cual intencionalmente carecía de exposición publicitaria. Es necesario considerar que los niños 
en la primera visita a la tienda no compraron todos los productos exhibidos, pero al finalizar el ejercicio todos los productos fueron los primeros en agotarse. Se destaca que cada niño invirtió un gran porcentaje de su dinero disponible en la realización de la compra de estos productos, pese a tener los precios más altos en relación con las demás categorías. En su orden, el primer producto en ser comprado fue el Shampoo, el cual contenía la imagen de Barbie para el caso de la línea femenina y de los Avengers para el caso de la línea masculina. Los últimos productos en agotarse correspondieron a los pañuelitos faciales que contenían motivos infantiles genéricos que no estaban respaldados por licenciaturas de marca.

Como segunda opción algunos niños fueron a la tienda de productos saludables y compraron en primera instancia Avena Alpina. Otros niños se dirigieron a la tienda de snacks y optaron por invertir su dinero en cereales para el desayuno Kellogg's. Paradójicamente, la última tienda en visitar fue la de golosinas donde el primer producto comprado correspondió a la marca Pony Malta; al finalizar el ejercicio el poco dinero sobrante fue invertido en los productos de menor valor individual, que para el caso correspondió a las gomas de mascar.

Los comportamientos observados permiten inferir que los niños no adquieren como primera opción los productos de las marcas expuestas en los videos. Por tanto, la televisión y el internet no constituyen una fuerte influencia en el consumidor de la base de la pirámide. En todos los niños investigados, su primera inversión giró en torno a los productos diseñados para el segmento infantil en la categoría de aseo personal.

Este comportamiento puede explicarse a partir de las respuestas que se obtuvieron cuando se realizaron las entrevista en profundidad, una vez finalizado el proceso de compra simulada. Se pudo identificar que en los hogares de todos los menores, las marcas correspondientes a la categoría de aseo personal son elegidas por los padres; quienes por su limitada capacidad de compra adquieren productos que funcionen para toda la familia, por ende, en su canasta están ausentes los productos especializados en el segmento infantil. Afirmaciones como "Eso yo solo lo había visto en televisión, nunca había tenido este champú en mis manos” evidencian estas carencias.

De otro lado, cuando se indagó por las razones que motivaron la compra de dulces y gomas de mascar sólo al final del proceso, los niños afirmaron que se trata de los productos que pueden adquirir a diario, por ello no les interesa hacer esta compra. "Es que un chicle lo puedo comprar cuando quiera”. Estas manifestaciones, reflejan los comportamientos hedo- 
nistas y utilitaristas de los consumidores desde temprana edad, sin distinguir el nivel socioeconómico al que pertenecen, pues se trata simplemente de un comportamiento humano.

Por lo anterior, se puede concluir que las necesidades básicas de los niños se encuentran satisfechas con productos para la familia, sin diferenciar los rangos etarios. De este modo, en el caso de los menores cuando disponen de dinero para realizar compras sin el control del adulto, prima sobre ellos un deseo insatisfecho que sacian cuando se presenta la oportunidad. Así pues, se cuestionan los argumentos relacionados con la influencia que ejercen los comerciales para promover el consumismo en los infantes. Sumado a esto, el estudio reveló que no es una constante el papel influenciador de los niños en los procesos de la compra de determinados productos o marcas, máxime cuando se trata de los consumidores de la base de la pirámide, donde el dinero es un factor determinante.

Para determinar la relación entre la influencia de la marca y el consumo, esta investigación pudo establecer que la mayor influencia corresponde a la dimensión meso de la marca donde involucra los rituales y los héroes. Según El-Amir (2010), "los rituales son actividades colectivas esenciales para una cultura, mientras que los héroes son personajes vivos o muertos, reales o hipotéticos, que poseen características muy apreciadas y por lo tanto representan un modelo de comportamiento". Para los niños la fuerza física y las actividades de acción se ven representadas en héroes de la firma Marvel; mientras que en el caso de las niñas, la belleza y la feminidad tienen como modelo representativo la muñeca Barbie de la firma Mattel.

\section{Influencia del precio}

Los niños que viven en el entorno de nivel socioeconómico más bajo de la ciudad, tienden a llamar de forma genérica al dinero "plata", bien sea para referirse a las monedas o a los billetes. Esta acepción puede estar soportada en la costumbre, puesto que para el caso colombiano, el material con el que antaño se acuñaron las monedas de baja denominación era la plata. En cuanto a la percepción del infante, la "plata" es considerada por el niño como el elemento que debe tener para realizar el proceso de compra de las "cosas". Se pudo identificar que estos consumidores carecen de la comprensión del concepto de precio desde el punto de vista técnico del marketing. 
Desde la perspectiva cualitativa, se observó que la influencia ejercida por el precio en los niños está relacionada con el tamaño del producto. Así, la mayoría de los niños considera que mientras más grande es un producto su precio es mayor; por el contrario, asociaron que los productos de tamaños pequeños tienen menores precios sin importar la categoría a que corresponda dicho producto. En la entrevista a profundidad se descubrió que tal situación se presenta a raíz de los "mandados". En este caso, cuando los niños son enviados a la tienda a realizar compras que en la mayoría de veces corresponden a productos relacionados con los alimentos, se debe cumplir la regla de la cantidad ligada al precio. Por ejemplo, en productos como el arroz, la leche o el pan mientras más grande es el producto mayor es su peso o su contenido, y por consiguiente mayor el precio que debe pagar para adquirirlo. Este proceso conlleva a que en su comprensión económica el niño (a) determine que si el dinero no alcanza para pagar el producto del "mandado", se compra uno de los mismos pero de menor tamaño. En los infantes del rango de edades de 5 a 6 años, la decisión se toma una vez ha preguntado al tendero “Esto para qué me alcanza?”, puesto que el vendedor le enseña artículos de menor precio pero de menor tamaño.

En la observación no participante, se evidenció este comportamiento cuando los pequeños se desplazaron a la tienda de aseo como primera opción de compra. Estos niños al inicio de la actividad contaron con COP50.000, los cuales representaban el total del dinero disponible cuando ingresaron a la tienda; allí eligieron los productos en tarro tipo champú o Gel que eran los más grandes y su precio oscilaba entre los COP18.000 y COP22.000. Llamó la atención que los niños en esa primera compra no tenían la capacidad matemática para realizar las operaciones de sumas y restas con billetes de altas denominaciones. No obstante, cuando llegaron al final del proceso con el billete de más baja denominación existente el Colombia, es decir COP 1.000, estos procesos mentales sí los realizaron de forma casi mecánica, tal como sucedió en la compra de dulces y gomas de mascar.

Al profundizar en la entrevista por este patrón de comportamiento, se encontró que para aquellos niños que tienen la opción de llevar dinero a la tienda, el valor máximo del que disponen es exactamente COP1.000. Además, a partir de sus respuestas, se pudo establecer que en términos generales los niños de la base de la pirámide tienen un contacto diario con todas denominaciones de las monedas, desde COP50 hasta COP1.000. Caso contrario sucede con los billetes, pues a medida que aumenta su denominación, es menos probable el manejo de billetes de sumas grandes en este segmento de consumidores. 


\section{Influencia y control parental}

Los niños de la base de la pirámide de Villavicencio no refieren control parental en temas asociados a las marcas de los productos disponibles en el mercado. El acompañamiento de los padres en su proceso de socialización con el dinero, y por consiguiente los controles que estos imponen a sus hijos, se remite a aspectos cotidianos. Por un lado, el sistema de pago se manifiesta principalmente en el crédito otorgado por la tienda del barrio, conocido como "el fiado". Por otro, la restricción en las compras queda circunscrita a la capacidad adquisitiva de la familia y del propio niño con las monedas que les son facilitadas, ello configura compras rutinarias de productos de bajo precio como los dulces, las gomas de mascar o las galletas. En el ejercicio de investigación a través de la simulación de la compra, estos productos fueron los últimos en ser adquiridos debido a que forman parte de su compra habitual; en tanto que los productos llamativos y con los cuales tienen un bajo o nulo contacto llamaron más la atención y fueron los más deseados por los niños, a tal punto que una vez agotado el dinero, algunos de estos infantes empezaron a proponer trueques con otros niños para satisfacer su deseo como consumidor.

En la entrevista a profundidad se indagó sobre las razones que motivaron la compra de los productos de aseo, pues como se mencionó, estos no se incluyeron en la publicidad. Los infantes afirmaron que nunca habían tenido un producto de esa categoría exclusivamente para ellos, además, manifestaron su desconocimiento en cuanto al uso de algunos de esos productos. Pero aun así, respondieron que los compraban para llevarlos a casa y poder compartirlos con sus hermanos.

Los resultados permiten inferir,que el control parental ejerce una escasa influencia en los infantes, y debido a la limitación en la comunicación familiar concretamente en temas de consumo, no es posible clasificar las familias según Rummel, Howard, Swinton \& Seymour (2000) o Herrera, Brito, Pérez, Martínez \& Díaz (2001).

\section{Influencia de los semejantes}

Durante la sesión de exposición publicitaria y compra de productos, se pudo evidenciar que los niños de 5 a 7 años se ven influenciados por los niños mayores. En algunas ocasiones 
optaron por seguirlos e imitarlos en su compra, principalmente en la primera tienda. No obstante, cuando descubrieron que tenían diferentes opciones en la oferta del mercado y cuando comprendieron que durante la actividad tenía libertad para seleccionar y pagar por el producto que querían, los menores iniciaron su proceso de forma independiente.

Un hallazgo que llamó la atención en estos niños, guarda relación con el proceso de transacción financiera, es decir, el pago por el producto. Estos pequeños, en múltiples oportunidades tomaron el producto y entregaron un billete sin determinar si realmente su valor alcanzaba para cubrir el precio; también se presentó el caso donde entregaron billetes de alta denominación para un producto de bajo precio, pero no esperaron el cambio. Por el contrario, los niños de 8 a 9 años expusieron con claridad sus deseos de consumo, pero racionalizaron la compra a través de la exposición de las necesidades que priman en su hogar. Además, asumieron el liderazgo y tomaron la iniciativa para empezar el proceso frente a las diferentes opciones que se presentaban en la simulación de los espacios comerciales.

Al realizar la entrevista a profundidad una vez finalizado el proceso, se les preguntó por la compra de determinados productos. Algunas expresiones de los niños fueron "de esto hablamos con mis amigos de la cuadra", "mi hermano me dice que él los ha visto" y "lo compro para probarlo con mis hermanitos". Esto demuestra que los niños con limitaciones económicas, responden de la misma forma que lo encontró Chikweche \& Fletcher (2011) para el entorno en Zimbabue, en el cual las marcas son reconocidas así no sean usadas. Esta investigación también se relaciona con el postulado de Dotson \& Hyatt (2000), en el sentido de que los niños que se quedan en casa sin supervisión de los padres, o aquellos hijos de padres o madres solteras tienden a recibir mayor influencia de sus semejantes representados en este caso en los amigos o hermanos. El mayor espacio de interacción con los semejantes es la escuela y la vecindad, puesto que realizan las compras en las cafeterías de los centros escolares o en las tiendas de barrio.

\section{Conclusiones}

Analizados los cuatro mecanismos de influencia en el comportamiento de compra de los niños de la base de la pirámide de Villavicencio (Colombia), se puede afirmar que estos se dividen en tres grupos: los influenciadores fuertes, los influenciadores moderados y final- 
mente, los influenciadores menores. Dentro del primer grupo, los influenciadores fuertes corresponden al precio de los productos y a los semejantes. La presente investigación no permite evidenciar cuál de ellos tendría mayor impacto en la decisión de los infantes, pero es claro que la capacidad adquisitiva y lo que refieren sus amigos o hermanos tiene una incidencia directa para la compra que realizan estos niños.

En el segundo grupo o influenciadores moderados se encuentra la marca de los productos. Pese a que los niños están expuestos a éstas y las reconocen, no tienen muchas posibilidades de utilizarlas porque no hacen parte de la canasta básica familiar comprada por los adultos. De este modo, las marcas de productos orientadas al segmento infantil ejercen menos presión para los consumidores de este nivel socioeconómico y para sus compradores adultos, quienes no se encuentran condicionados por el entorno para hacer uso de ellas. En el último grupo de los influenciadores menores se encuentra el control parental. A partir de las condiciones laborales de los adultos pertenecientes a este nivel socioeconómico que en la mayoría de casos está ligada a la informalidad, sumado a las extensas jornadas de trabajo y a la permanencia fuera de casa durante el día, se pudo establecer la escasa relación. Esto se debe a que existe un bajo contacto de socialización económica con sus hijos, lo que conlleva a que su influencia en las decisiones de los más pequeños sea mínima.

Sin lugar a dudas, atender el mercado de los consumidores infantiles de la base de la pirámide exige la comprensión por parte de los empresarios y personas de las ciencias empresariales. Estos niños desean productos segmentados, pero sus padres o adultos responsables no tienen la liquidez necesaria para comprarlos. Adicionalmente, estos consumidores reconocen las marcas existentes y disponibles en el mercado pero debido a la restricción presupuestal no ejercen una influencia fuerte. Por último, se evidencia un direccionamiento marcado en la decisión de compra a través de los semejantes, lo que configura a las escuelas y tiendas de barrio como puntos de referencia muy importantes para desarrollar las estrategias de marketing en el segmento estudiado.

\section{Restricciones y futuras líneas de investigación}

La presente investigación tomó en cuenta niños (a) estudiantes de instituciones públicas de la ciudad, por ello los resultados de las influencias no podrían ser extrapolados a la pobla- 
ción de la misma base de la pirámide pero en condición de desescolarización. Esto se debe a que dentro de las conclusiones del estudio aparece la escuela como elemento de interacción con los semejantes y lugar de compra; lo cual no es posible afirmarlo en los infantes que actualmente no se encuentran estudiando. También se plantea como restricción que solo se analizaron cuatro influenciadores, sin considerar otras fuerzas importantes como los medios masivos de comunicación.

Asimismo, se hace referencia que durante el desarrollo de la investigación, resulto evidente la escasa disponibilidad de estudios relacionados con el análisis sobre el comportamiento de consumidores del segmento infantil en Villavicencio -Colombia, de tal manera que la búsqueda exhaustivas sobre publicaciones en el área determinó que el comportamiento de estos consumidores pertenecientes a la clase social menos favorecida amerita ser considerada como un referente para futuras investigaciones, por lo que como futura línea de investigación se plantea el uso de los medios masivos de comunicación y su influencia en este segmento de consumo.

\section{Agradecimientos}

Los autores del presente artículo agradecen a la profesora Angélica Sofía González Pulido por su participación en la realización del trabajo de campo de esta investigación.

\section{Referencias}

Acevedo, C (2005). Relativismo y marketing: Una propuesta metodológica para el estudio del comportamiento del consumidor. Pensamiento \& Gestión, 19, 1-42.

Amar, J.; Llanos, M.; Abello, R. y Denegri, M. (2003). Desarrollo del pensamiento económico en niños de la región caribe colombiana. Revista Latinoamericana de Psicología, 35 (1), 7-18.

Báez, P. J (2012). Investigación cualitativa, México: Alfaomega y Madrid, España: Esic.

Chikweche, T. \& Fletcher, R (2011). Branding at the base of pyramid: a Zimbabwean perspective. Marketing Intelligence \& Planning, 29 (3), 247-263. 
Cook, D.T (2009). Knowing the child consumer: historical and conceptual insights on qualitative children's consumer research. Young Consumers, 10 (4), 269-282.

Creswell, J. (1994). Diseño de la investigación. Enfoques cualitativos y cuantitativos. Cátedra de Ciencias Sociales.

Delval, J. y Echeita, G (1991). La comprensión en el niño del mecanismo de intercambio económico y el problema de la ganancia. Revista Infancia y Aprendizaje, 54, 71-100.

Dotson, M.J. \& Hyatt, E.M. (1994). The impact of changes in the household on the consumer socialization process. Proceedings of the Southern Marketing Association, New Orleans, USA.

Dotson, M.J. \& Hyatt, E.M (2000). A comparison of parents' and children's knowledge of brands and advertising slogans in the United States: implications for consumer socialization. Journal of Marketing Communications, 6 (4), 219-30.

El-Amir, A. \& Burt, S (2010). Modeling in branding: a critical ethnography approach. Qualitative Market Research: An International Journal, 13 (2), 189- 209.

Fernández, J. P. (2008, 8 de enero). Nadie puede innovar por sí solo. Dinero. Recuperado de http://www.dinero.com/caratula/edicion-impresa/articulo/nadie-puede-innovar-solo/65994

Herrera, E. Brito, A. Pérez, J. Martínez, M. \& Díaz, A (2001). Percepción de estilos educativos parentales e inadaptación en adolescentes. Revista de Psicología Universitas Tarraconensis, 23 (1-2), 44-57.

Kotler, P. \& Armstrong, G (2012). Marketing, México: Pearson Educación.

Mendoza, F. D. \& López, J. D (2015). Pensamiento estratégico: centro neurálgico de la planificación estratégica que transforma la visión en acción. Revista Económicas CUC, 36 (1), 153-179.

McNeal, J.U (1987). Children as consumers: insights and implications, Lexington, USA: D.C. Heath and Company.

Mocan, N (2007). Can consumers detect lemons? An empirical analysis of information asymmetry in the market for child care. Journal of Population Economics, 20 (4), 743-780. 
Moschis, G. \& Churchill, G (1978). Consumer socialization: a theoretical and emprical analysis. Journal of Marketing Research, 15, 599-609.

Moschis, G. \& Moore, R.L (1979), Decision making among the young: a socialization perspective. Journal of Consumer Research, 6, 101-12.

Nairn, A., Griffin, C. \& Wicks G.P (2008). Children's use of brand symbolism: A consumer culture theory approach. European Journal of Marketing, 42, 627-640.

Otero, M.C. y Giraldo, W (2015). El consumidor infantil en Villavicencio, Colombia. Criterio Libre, 13 (22), 173-197.

Prahalad, C.K (2005). La oportunidad de negocios en la base de la pirámide, Bogotá, Colombia: Grupo Editorial Norma.

Paul, N.C. (2002, 1 de abril). Branded for life? The Christian Science Monitor. Recuperado de ttp://www.csmonitor.com/2002/0401/p15s02-wmcn.html

Portela, L. (2011). Influencia de los niños en la decisión de compra de los padres. Estudio exploratorio con niños de 6 a 9 años de edad y padres de familia de la ciudad de Santiago de Cali. (Tesis de pregrado). Universidad Autónoma de Occidente, Cali, Colombia.

Reyes, R. D (2013). La etnografía en los estudios de marca: una revisión bibliográfica. Pensamiento \& Gestión, 34, 211-234.

Roper, S., \& Shah, B (2007). Vulnerable consumers: the social impact of branding on children. Equal Opportunities International, 26 (7), 712-728.

Rummel, A., Howard, J., Swinton, J. M., \& Seymour, D. B (2000). You can't have that! A study of reactance effects \& children's consumer behavior. Journal of Marketing Theory and Practice, 8 (1), 38-45.

Silva, A.E. (2009). El consumo, ¿un juego de niños? Cuadernos Latinoamericanos de Administración, 5 (9), 67-82.

Tur, V. y Ramos, I (2008). Marketing y niños, Madrid, España: ESIC Editorial.

Villalba, B. M., Hurtado, B. H., Guarín, R. H. \& Casas, O. J (2013). Innovación en pymes artesanales de Morroa, Sucre, 2012. Revista Económicas CUC, 34 (1), 15-28.

Wolf, M (1982). Sociologías de la vida cotidiana, Madrid, España: Ediciones Cátedra. 